\title{
Beam breakup instability in an annular electron beam
}

\author{
Y. Y. Lau and John W. Luginsland \\ Intense Energy Beam Interaction Laboratory and Department of Nuclear Engineering, \\ University of Michigan, Ann Arbor, Michigan 48109-2104
}

(Received 8 March 1993; accepted for publication 19 July 1993)

It is shown that an annular electron beam may carry six times as much current as a pencil beam for the same beam breakup (BBU) growth. This finding suggests that the rf magnetic field of the breakup mode is far more important than the rf electric field in the excitation of BBU. A proof-of-principle experiment is suggested, and the implications explored.

Annular electron beams have the capability of carrying a much higher current than a pencil beam. Besides the obvious fact that annular beams have a larger crosssectional area, their limiting currents are significantly higher than those of a pencil beam when placed in a metallic drift tube. For this and other reasons, annular beams have recently been chosen as the preferred geometry to generate coherent, ultrahigh power microwaves. ${ }^{1,2}$ They have also been used as the primary beam in several "twobeam accelerator" configurations. ${ }^{3,4}$ These annular beams either encounter a sequence of modulating gaps, or simply glaze by a slow wave structure to generate a wake field in the case of two-beam accelerators. ${ }^{3}$ The beam radius, the pill box radius, and the slow wave structure radius may all be of the same order of magnitude. The high current would then lead to the beam breakup instability $(B B U)^{5-8}$ and this concern motivates the present study.

$\mathrm{BBU}$ is usually analyzed for a pencil beam propagating along the center axis of a sequence of accelerating cavities. Many BBU calculations of practical interest assume that the accelerating unit is the familiar cylindrical pillbox cavity and that the dominant deflecting mode is the $T M_{110}$ mode. ${ }^{5,6,10}$ Extension to an annular beam is straightforward. Nevertheless, this calculation leads to several unexpected results and provides some new insights into BBU, to be reported in this communication.

It is well known that BBU is excited by the combined action of the $\mathrm{rf}$ magnetic field $\left(\mathbf{B}_{1}\right)$ and the $\mathrm{rf}$ electric field $\left(\mathbf{E}_{1}\right)$ of the deflecting modes: ${ }^{5} \mathbf{B}_{1}$ causes beam deflection through the Lorentz force and $\mathbf{E}_{1}$ causes mode amplification through the work done on the mode by the beam current $\mathbf{J}$. Our calculation strongly suggests that $\mathbf{B}_{1}$ is much more critical than $\mathbf{E}_{1}$ in contributing to $\mathrm{BBU}$ growth. Thus, an annular beam strategically placed near the minimum of the if magnetic field would suffer far less beam breakup growth than a pencil beam that is centered on the cavity axis, where the magnetic field is large and the axial electric field is small. By the same argument, placing the annular beam very close to the wall of a metallic drift tube, at which the axial electric field is vanishingly small, cannot eliminate BBU growth because of the substantial deflecting magnetic field generated by the wall current. Toward the end of this communication, we propose an experiment which would unambiguously test the relative importance between the rf magnetic field and the $\mathrm{rf}$ axial electric field, as discussed here.
Consider an infinitesimally thin annular beam of radius $r_{0}$ inside a cylindrical pillbox of radius $b$. The beam carries a total current $I$ and coasts at velocity $v_{0}$ with the corresponding relativistic factors $\gamma$ and $\beta$. The drift tube is loaded with a slow wave structure, modeled by a series of cylindrical pillbox cavities, each of which supports the nonaxisymmetric $\mathrm{TM}_{110}$ mode. ${ }^{3,5,6,10}$ The interaction between this mode and the beam causes BBU to be excited. In the limit $r_{0} \rightarrow 0$, this is the basic model of BBU for a pencil beam. Since we are comparing the strength of BBU interaction for different values of $r_{0}$, we pretend that magnetic focusing is absent and that the quality factor $Q$ of the deflecting mode is infinite.

Let $\mathrm{A}_{1}=\hat{z} q(t)(\cos \theta) E(r)$ be the vector potential of the deflecting dipole mode in a cavity. For the fundamental $\mathrm{TM}_{110}$ mode, $E(r)=J_{1}(p r)$ represents the radial dependence of the axial electric field with $J_{1}$ being the Bessel function of order one and $p=3.832 / b$. The corresponding magnetic field is $\mathbf{B}_{1}=\nabla \times \mathbf{A}_{1}$. The action of this mode on the beam is calculated as follows.

We divide the annular beam into $N$ azimuthal segments ( $N$ large). The $i$ th segment is located at $r=r_{0}$, $\theta=\theta_{i}=2 \pi i / N$, in the unperturbed state but is displaced radially by $\xi_{i}$ and azimuthally by $\eta_{i}$ when the deflecting mode is present. The linearized force law yields

$$
\begin{aligned}
-\gamma\left(\omega-k v_{0}\right)^{2} \xi_{i}= & \left(e / m_{0}\right)\left(v_{0} / c\right) q E^{\prime}\left(r_{0}\right) \cos \theta_{i}, \\
-\gamma\left(\omega-k v_{0}\right)^{2} \eta_{i}= & -\left(e / m_{0}\right) \\
& \times\left(v_{0} / c\right) q\left[E\left(r_{0}\right) / r_{0}\right] \sin \theta_{i},
\end{aligned}
$$

where the right-hand sides represent the components of the Lorentz force that causes beam deflection. In writing Eqs. (1) and (2), we have assumed a wave-like solution $\exp [j(\omega t-k z)]$ for the disturbances, with $j^{2}=-1$, and we have used a prime to denote derivative with respect to the argument.

The instantaneous current $J$ on the $i$ th current filament is

$$
\mathbf{J}_{i}(r, t)=\hat{z} \frac{I}{N} \frac{1}{r} \delta\left(r-r_{0}-\xi_{i}\right) \delta\left(\theta-\theta_{i}-\frac{\eta_{i}}{r_{0}}\right)
$$

where $\delta$ is the Dirac delta function. The work done by this current filament on the deflecting mode is proportional to

$$
W_{i}=\int d V \mathbf{A}_{1} \cdot \mathbf{J}_{i}
$$




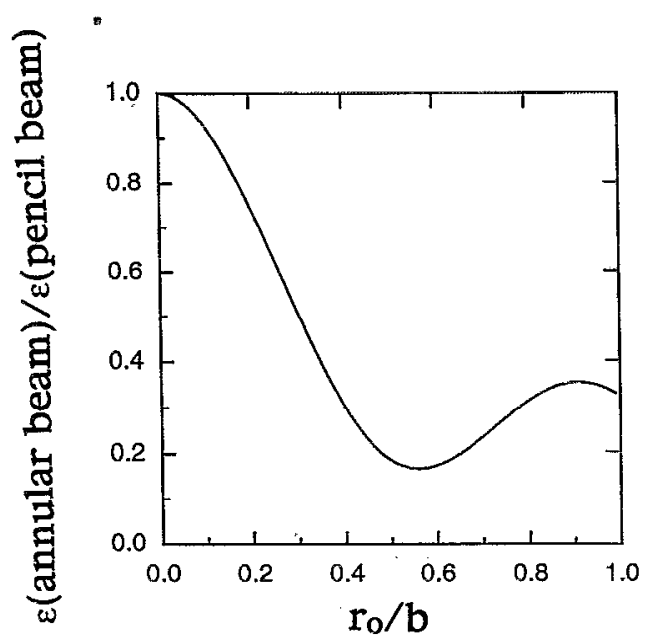

FIG. 1. Comparison of the BBU coupling constant $\epsilon$ between an annular beam of radius $r_{0}$ and an on-axis pencil beam $\left(r_{0} \rightarrow 0\right)$ with the same total current.

where the volume integral is performed over the cavity. In evaluating $W_{i}$, we should retain only the rf component of $\mathbf{J}_{i}$ in Eq. (3), since only the rf current performs work on the breakup mode. Upon substituting Eqs. (1)-(3) into Eq. (4), and summing over all $i$, we find the total work done

$W=\sum_{i=1}^{N} W_{i}=-\frac{L I e}{m_{0}} \frac{v_{0}}{c} q \frac{\left[E^{\prime}\left(r_{0}\right)\right]^{2}+\left[E\left(r_{0}\right) / r_{0}\right]^{2}}{\gamma\left(\omega-k v_{0}\right)^{2}}$

apart from a multiplicative constant that is independent of the beam's equilibrium position $r_{0}$. This energy transfer leads to growth of the BBU mode, which is described by the BBU dispersion relation: ${ }^{8}$

$$
\left(\omega^{2}-\omega_{0}^{2}\right)\left(\omega-k v_{0}\right)^{2}=-2 \omega_{0}^{4} \epsilon=-2 \omega_{0}^{4} \epsilon_{0}\left(\epsilon / \epsilon_{0}\right),
$$

where $\epsilon$ is the coupling constant and $\omega_{0}$ is the breakup mode frequency. In writing the last form of Eq. (6), we normalize $\epsilon$ in terms of $\epsilon_{0}$, the coupling constant for an on-axis, pencil beam $\left(r_{0} \rightarrow 0\right)$. For the $\mathrm{TM}_{110}$ mode, $E=J_{1}(p r)$ and $\epsilon_{0}=0.422(\beta / \gamma)(I / 1 \mathrm{kA})$. It is clear from Eq. (5) that

$$
\frac{\epsilon}{\epsilon_{0}}=2\left\{\left[J_{1}^{\prime}\left(p r_{0}\right)\right]^{2}+\left[\frac{J_{1}\left(p r_{0}\right)}{p r_{0}}\right]^{2}\right\},
$$

which compares the BBU strength between an annular beam and a pencil beam of the same current. Note that this ratio reduccs to unity in the limit $r_{0} \rightarrow 0$.

Equation (7) is plotted in Fig. 1 as a function of $r_{0} / b$. It is seen from this figure that $\epsilon / \epsilon_{0}$ may be as small as 0.17 when the annular beam is located at $r_{0}=0.56 \mathrm{~b}$. Note also that this location coincides with the minimum of the rf magnetic field of the deflecting mode. What this means is that an annular beam placed at this location can carry as much as $1 / 0.17=6$ times the current as an on-axis pencil beam, and suffer the same BBU growth. Another point worth noting is that BBU growth retains significant strength even if the annular beam is very close to the wall of the drift tube (cf. $r_{0} \rightarrow b$ in Fig. 1). This result is unex- pected since $\mathbf{E}_{1} \rightarrow 0$ near a metallic wall. As a result, $\mathbf{J}_{1} \cdot \mathbf{E}_{1} \rightarrow 0$ and, superficially, one could hardly expect any transfer of power from the beam to drive the breakup mode. ${ }^{9}$ The finite BBU strength as $r_{0} \rightarrow b$ is another strong indication that the deflecting magnetic field is far more important than the axial rf electric field in driving BBU.

The importance of the rf magnetic field can be tested in an experiment in which a pencil beam is focused by a solenoidal magnetic field and is made to pass through a sequence of pillbox cavities, in which the first cavity is primed with microwaves at the $\mathrm{TM}_{110}$ mode. ${ }^{10} \mathrm{BBU}$ growth is monitored at the last cavity, before the beam exit. The above theory then predicts the unusual feature that BBU growth should be much less if the pencil beam is placed off-axis, than if the pencil beam were on-axis. ${ }^{11}$ The BBU growth should be minimum if this pencil beam is placed at a distance of about 0.56 of the pillbox radius, where the rf magnetic field is minimum.

We also repeated the calculations for the higher order radial modes: $\mathrm{TM}_{120}, \mathrm{TM}_{130}, \mathrm{TM}_{140}$, and $\mathrm{TM}_{150}$. Fixing $r_{0} / b=0.56$, the ratio $\epsilon / \epsilon_{0}$ equals $0.16,0.012,0.037$, and 0.013 for these four higher order modes, respectively. Thus, the annular beam still suffers substantially lower BBU growth, in the higher order deflecting modes, than an on-axis pencil beam of the same current.

In conclusion, the if magnetic field is found to be much more important than the if electric field in contributing to BBU growth. A simple proof-of-principle experiment is proposed to test this new finding. Annular beams are far more stable than an on-axis pencil beam, as a result.

We thank Professor Ronald Gilgenbach for his support and for many useful discussions. This work was supported in part by an SDIO/IST contract managed by ONR.

${ }^{1}$ V. Serlin and M. Friedman, Appl. Phys. Lett. 62, 2772 (1993); Y. Y. Lau, M. Friedman, J. Krall, and V. Serlin, IEEE Trans. PS-18, 553 (1990) and references therein; M. Friedman, Y. Y. Lau, J. Krall, and V. Serlin, U. S. Patent 5,132,638 (issued July 21, 1992); J. Krall, M. Friedman, Y. Y. Lau, and V. Serlin, IEEE Trans. EMC-34, 22 (1992).

${ }^{2}$ C. Chen, P. Catraras, and G. Bekefi, Appl. Phys. Lett. 62, 1579 (1993); See also, "Intense Microwave and Particle Beams III," Proc. Soc. Photo Opt. Instrum. Eng. SPIE 1629 (1992).

${ }^{3} \mathrm{G}$. Voss and T. Weiland, "The wakefield acceleration mechanism," Deutsches Electron-Synchrotron Internal Report DESY \#M82-10 (1982); M82-079 (1982), Hamburg, Germany.

${ }^{4}$ M. Friedman, J. Krall, Y. Y. Lau, and V. Serlin, Phys. Rev. Lett. 63, 2468 (1989).

${ }^{5}$ W. K. H. Panofsky and M. Bander, Rev. Sci. Instrum. 39, 206 (1968);

R. H. Helm and G. A. Loew, in Linear Accelerators, edited by R. P. Lapostolle and A. L. Septier (North-Holland, Amsterdam, 1970), p. 173.

${ }^{6}$ V. K. Neil, L. S. Hall, and R. K. Cooper, Part. Accel. 1, 111 (1970); 9, 213 (1979).

${ }^{7}$ A. W. Chao, B. Richter, and C. Y. Yao, Nucl. Instrum. Methods 178, 1 (1980); K. A. Thompson and R. D. Ruth, Phys. Rev. D 41, 964 (1990); R. L. Gluckstern, F. Neri, and R. K. Cooper, Part. Accel. 23, 37 (1988); C. L. Bohn and J. R. Delayen, Phys. Rev. A 45, 5964 (1992); D. Chernin and A. Mondeli, Part. Accel. 24, 685 (1985); G. Decker and J. M. Wang, Phys. Rev. D 38, 980 (1988); W. E. Martin, G. J. Caporaso, W. M. Fawley, D. Prosnitz, and A. G. Cole, Phys. Rev. Lett. 54, 685 (1985); D. Colombant, Y. Y. Lau, and D. Chernin, Part. Accel. 35, 193 (1991).

${ }^{8}$ Y. Y. Lau, Phys. Rev. Lett. 63, 1141 (1989). 
${ }^{9}$ The rate of power transfer is proportional to the gradient of $\mathbf{E}_{1}$, rather than $\mathbf{E}_{1}$ itself. This may be seen when one substitutes Eq. (3) into Eq. (4) and performs integration by parts.

${ }^{10}$ P. R. Menge, R. M. Gilgenbach, and Y. Y. Lau, Phys. Rev. Lett. 69, 2372 (1992); P. R. Menge, R. M. Gilgenbach, and R. Bosch, Appl. Phys. Lett. 61, 642 (1992).
${ }^{11} \mathrm{BBU}$ growth on a pencil beam that is placed off-center can be easily calculated by using Eq. (4) instead of Eq. (5). We pretend that the total beam current is carried by the $i$ th filament that enters Eq. (4). Although the BBU growth of such an off-center beam depends on $\theta_{i}$, its coupling constant $\epsilon$ is still much less than $\epsilon_{0}$, the value for an on-axis beam. 